\title{
¿CÓMO MINISTRAR A LOS HOMOSEXUALES?
}

\author{
Ekkehardt Mueller
}

\section{Introducción}

La homosexualidad se ha vuelto un tema extremadamente controversial en muchos países alrededor del mundo, impactando a varias sociedades y comunidades cristianas. ¿Qué se debería hacer si un miembro de iglesia se siente atraído por alguien del mismo sexo? ¿Cómo se deberían evaluar sus inclinaciones? ¿Cómo deberían los heterosexuales, así como la iglesia y el pastor, tratar con personas que practican la homosexualidad y/o promueven un estilo de vida homosexual? ¿Cómo deberían los administradores de la iglesia tratar con empleados que se identifican como homosexuales activos? ¿Qué debería hacer la administración de la iglesia en el caso de que el gobierno trate de obligar a que se empleen personas homosexuales? Pero la pregunta que está detrás de todas estas es: ¿Cómo se relacionan los cristianos con la Biblia? Por estas preguntas algunas denominaciones corren el riesgo de dividirse o ya se han dividido sobre este asunto. ${ }^{1}$

Nuestra investigación no se enfocará en estudiar los textos de la Biblia que tratan directa o indirectamente con

*Una vez más, quiero agradecer a Joel Iparraguirre por su gran trabajo al haber adaptado y traducido este artículo.

${ }^{1}$ Véase Andreas J. Köstenberger, God, Marriage, and Family: Rebuilding the Biblical Foundation (Wheaton: Crossway, 2004), 202; Craig L. Nessan, Many Members, One Body: Committed Same-Gender Relationships and the Mission of the Church (Minneapolis: Fortress Press, 2004). 
la cuestión de la homosexualidad. Estos ya han sido analizados en otros documentos, ${ }^{2}$ así que el objetivo principal de este artículo es sugerir, tanto a los miembros de iglesia como a los pastores, algunas pautas que podrían ayudar al momento de tratar con personas homosexuales. Sin embargo, antes de ello, será necesario (1) definir qué es la homosexualidad, (2) conocer las diversas interpretaciones que se han dado sobre este tema, (3) notar si Jesús dijo algo sobre la homosexualidad y cuál fue su actitud, y (4) mostrar la posición de la Iglesia Adventista sobre este peculiar tema.

\section{Definiendo la homosexualidad}

La homosexualidad ha sido entendida de diferentes maneras, pero usualmente es descrita como "el deseo sexual hacia personas del mismo sexo". ${ }^{3}$ También puede designar a una "persona, varón o mujer, que en su vida adulta experimenta una atracción erótica estable y casi exclusiva hacia miembros del mismo sexo, y que es indiferente a las relaciones sexuales con el sexo opuesto". ${ }^{4}$ Una definición tal encaja con los "homosexuales constitucionales" o "invertidos" cuya homosexualidad supuestamente es permanente. También hay casos de adolescentes cuya

${ }^{2}$ Para mayor información, véase Ekkehardt Mueller, Homosexualidad, la Biblia y la iglesia, trad. Joel Iparraguirre (Ciudad de México: GEMA Editores, 2016); Ronald M. Springett y Ekkehardt Mueller, La homosexualidad en la historia y en la Biblia, trad. Joel Iparraguirre y Eric E. Richter (Ciudad de México: GEMA Editores, 2017).

${ }^{3}$ R. E. O. White, "Homosexuality", en Evangelical Dictionary of Theology, ed. Walter A. Elwell (Grand Rapids, MI: Baker, 1986), 528.

${ }^{4}$ E. A. Malloy, Homosexuality and the Christian Way of Life (Lanham, MD: University Press of America, 1981), 11. 
identidad sexual no se ha desarrollado completamente o adultos que están aburridos con la heterosexualidad y que están dispuestos a experimentar con miembros del mismo sexo. Ellos son llamados "homosexuales contingentes". Los "homosexuales situacionales" son aquellos que, debido a la falta de encuentros heterosexuales, recurren a citas o encuentros homosexuales. ${ }^{5}$

Normalmente, los "invertidos" afirman que su homosexualidad es innata, natural e irreversible. ${ }^{6}$ La distinción entre la orientación homosexual y los actos homosexuales usualmente es rechazada por la comunidad homosexual. ${ }^{7}$ Aun así, la orientación homosexual es reconocida por la iglesia y requiere una vida de celibato, mientras que la práctica homosexual es rechazada. Los actos homosexuales pueden expresarse, desde un punto de vista bíblico,${ }^{8}$ en la pederastia, la participación con niños del mismo sexo, las violaciones y violencia, prostitución y promiscuidad, por nombrar algunas prácticas, o en una vida comprometida con una pareja del mismo sexo. Algunos afirman que esta última opción está en armonía con la Escritura.

${ }^{5}$ Ronald M. Springett, Homosexuality in History and the Scriptures (Silver Spring, MD: Biblical Research Institute of the General Conference, 1988), 2.

${ }^{6}$ Véase Jack Rogers, Jesus, the Bible, and Homosexuality: Explode the Myths, Heal the Church (Louisville, KY: Westminster John Knox Press, 2009), 79; Aubyn Fulton, "Response; Science and Sexual Orientation", en Christianity and Homosexuality: Some Seventh-day Adventist Perspectives, ed. David Ferguson, Fritz Guy y David R. Larson (Roseville, CA: Adventist Forum, 2008), parte 2 - 47.

${ }^{7}$ Springett, Homosexuality, 4.

${ }^{8}$ Véase, por ejemplo, Ekkehardt Mueller, "Same Sex Sexual Relations in Romans 1:26-27" (Documento inédito, Biblical Research Institute, 2017). 


\section{Diversidad de interpretaciones}

La interpretación de la Biblia depende hasta cierto grado de ciertas presuposiciones. La manera en que el individuo ve a la Escritura, la cultura, la ciencia, la tradición y la naturaleza humana influye en su acercamiento a la Biblia.

\section{Diversas presuposiciones}

El punto de vista prevaleciente entre los pensadores de las sociedades occidentales es que no hay ninguna verdad absoluta, que no hay ninguna revelación divina, y que las revisiones y reformulaciones de las antiguas creencias son necesarias para que encajen en la cultura prevaleciente. ${ }^{9}$ Se considera que la Biblia está culturalmente condicionada, es decir, que ha hablado solo a ciertas situaciones en el pasado pero debe ser reinterpretada en la actualidad. ${ }^{10} \mathrm{Se}$ mantiene que "nuestra cosmovisión moderna incluye avances y descubrimientos desconocidos para las personas antiguas, haciendo que los pronunciamientos bíblicos sobre la homosexualidad sean incompletos e incluso erróneos". ${ }^{11}$ Por

9Véase Sheila Greeve Davaney, Historicism: The Once and Future Challenge for Theology; Guides to Theological Inquiry (Minneapolis: Fortress Press, 2006), 160-4. Walter Wink sostiene que "cuando la Biblia menciona los comportamientos homosexuales, claramente los condena. Puedo garantizar eso libremente. El asunto es precisamente si el juicio bíblico es correcto". Walter Wink, "Homosexuality and the Bible", en Homosexuality and Christian Faith: Questions of Conscience for the Churches, ed. Walter Wink (Minneapolis: Fortress Press, 1999), 47. Cf. Daniel A. Helminiak, What the Bible Really Says About Homosexuality (New Mexico: Alamo Square Press, 2000), 131.

${ }^{10} \mathrm{Cf}$. Wink, "Homosexuality and the Bible", 35, 42; Rogers, Homosexuality, 69, 70; Webb, 161.

${ }^{11}$ James B. de Young, Homosexuality: Contemporary Claims Examined in the Light of the Bible and Other Ancient Literature and Law (Grand Rapids, MI: Kregel Publications, 2000), 11. Véase también Springett, Homo- 
lo tanto, la propia comprensión de la cultura greco-romana determina cómo los textos del NT deben ser interpretados. ${ }^{12}$ Se dice que "la Biblia se opone a la homosexualidad cuando está relacionada a la prostitución y a la idolatría, pero no a la homosexualidad en sí". ${ }^{13}$ También se ha sugerido que la Escritura no aborda las relaciones monógamas y permanentes del mismo sexo ${ }^{14}$ porque supuestamente no está al tanto de la existencia de la homosexualidad innata o invertida, ${ }^{15} \mathrm{y}$ que solo se refiere a la homosexualidad abusiva, tales como la pederastia, ${ }^{16}$ las violaciones, la promiscuidad, o el exceso de pasión. ${ }^{17}$

Algunos eligen el "principio cristológico" y rechazan las declaraciones bíblicas que parecen contradecirlo. ${ }^{18}$ Esto

sexuality, 49-51; Marion L. Soards, Scripture and Homosexuality: Biblical Authority and the Church Today (Louisville, KY: Westminster John Knox Press, 1995), 55 .

${ }^{12}$ Cf. Robin Scroggs, The New Testament and Homosexuality (Minneapolis: Fortress Press, 1983), 16, 127, 128.

${ }^{13}$ Springett, Homosexuality, 51, aunque esta no es su propia posición. Gary Chartier, "Love, Subsidiarity, Equality, and Inclusiveness", en Christianity and Homosexuality, parte 5 - 58

${ }^{14}$ Véase Springett, Homosexuality, 50; Vincent J. Genovesi, In Pursuit of Love: Catholic Morality and Human Sexuality, 2da ed. (Collegeville, MN: Liturgical Press, 1996), 277, 296; Ellen F. Davis, "Reasoning with Scripture", Anglican Theological Review 90, no. 3 (2008): 518; Rogers, Homosexuality, 89.

${ }^{15} \mathrm{Cf}$. Scroggs, The New Testament and Homosexuality, 28.

${ }^{16}$ Véase ibíd., 84.

${ }^{17} \mathrm{Cf}$. David E. Fredrickson, "Natural and Unnatural Use in Romans 1:24-27: Paul and the Philosophic Critique of Eros", en Homosexuality, Science, and the "Plain Sense" of Scripture, ed. David L. Balch (Grand Rapids, MI: Eerdmans, 2000), 197-222.

${ }^{18} \mathrm{Cf}$. Rogers, Homosexuality, 15, 53-5, 66. Véase también Wink, "Homosexuality and the Bible", 47, 48. William Sloane Coffin indica que "no todo 
quiere decir que debido a que Jesús hubiese aceptado a los que practican actos homosexuales, nosotros deberíamos hacer lo mismo, independientemente de cualquier declaración bíblica que indique lo contrario. ${ }^{19}$ Esto, por lo tanto, significaría que la iglesia, movida por el Espíritu Santo, sería libre de aceptar o rechazar las leyes bíblicas ${ }^{20}$ y que la autoridad de la Escritura estaría seriamente restringida. ${ }^{21}$ Otros van un paso más allá, afirmando que siguen al Espíritu Santo individualmente, incluso si sus conclusiones contradicen la Escritura. ${ }^{22}$

Algunos enfrentan la ley contra el evangelio. ${ }^{23}$ Se dice que el concepto del amor supera las interpretaciones estrictas de textos bíblicos. Otros consideran que sus experiencias personales son normativas y las usan para rechazar o

lo bíblico es cristiano”. William Sloane Coffin, "Liberty to the Captives and Good Tidings to the Afflicted", en Homosexuality and Christian Faith, 107.

${ }^{19} \mathrm{Cf}$. Nancy Duff, "Christian Vocation, Freedom of God, and Homosexuality”, en Homosexuality, Science, 261-77.

${ }^{20} \mathrm{Cf}$. Soards, Scripture and Homosexuality, 17; Wink, "Homosexuality and the Bible", 42-4.

${ }^{21}$ Richard Treloar, “'Come Out and Stay Out!' Hermeneutics, Homosexuality, and Schism in Anglicanism”, Anglican Theological Review 90, no. 1 (2008): 54, 55. Richard Treloar añade que "los anglicanos pueden resistir la 'enseñanza clara' de la Biblia en este asunto, como ya lo hacemos patentemente acerca de muchas otras cosas... 'con' la Escritura... a veces debemos leer 'en contra' de la Escritura”. Ibíd., 58. "La Biblia... no es directamente el equivalente de la palabra de Dios...” Ibíd., 61.

${ }^{22}$ Cf. James A. Forbes Jr., "More Light from the Spirit on Sexuality", en Homosexuality and Christian Faith, 6-8; Ken Sehested, "Biblical Fidelity and Sexual Orientation: Why the First Matters, Why the Second Doesn't", en Homosexuality and Christian Faith, 59; Richard Rohr, "Where the Gospel Leads Us", en Homosexuality and Christian Faith, 85-8.

${ }^{23}$ Marion L. Soards declara: "La gracia, no la ley, gobierna la vida cristiana”. Soards, Scripture and Homosexuality, 17. 
aceptar declaraciones bíblicas específicas. ${ }^{24}$ Otros le dan prioridad a la ciencia, las humanidades y la razón antes que a la Escritura ${ }^{25}$ o sugieren que tenemos que usar, como autoridad final, a la Escritura más la ciencia/razón (e.g., la biología, la sociología y la psicología) ${ }^{26}$ la tradición ${ }^{27}$ y la experiencia para tomar decisiones informadas. ${ }^{28}$ Además, se asume que el impulso sexual humano debe ser expresado ya

${ }^{24} \mathrm{Cf}$. Paul Wennes Egertson, “One Family's Story”, en Homosexuality and Christian Faith, 23-30; Phyllis A. Bird, "The Bible in Christian Ethical Deliberation Concerning Homosexuality: Old Testament Contributions", en Homosexuality, Science, 143; John B. Cobb Jr., "Being Christian about Homosexuality", en Homosexuality and Christian Faith, 91-3; René D. Drumm, "Interaction and Angst: The Social Experiences of Gay and Lesbian Seventh-day Adventists", en Christianity and Homosexuality, parte 3 - 20.

${ }^{25}$ Bird, 168; Wink, "Homosexuality and the Bible", 46; Rogers, Homosexuality, 35,36 .

${ }^{26} \mathrm{Cf}$. Ben Kemena, "Biological Determinants of Homosexual Orientation", en Christianity and Homosexuality, parte 2 - 16-19; Harry C. Wang, "Psychiatry, Antihomosexual Bias, and Challenges for Gay and Lesbian Youth", en Christianity and Homosexuality, parte 2 - 40; Fulton, "Response; Science and Sexual Orientation", parte 2 - 48, 49; Sherwood O. Cole, "Biology, Homosexuality, and the Biblical Doctrine of Sin”, Bibliotheca Sacra 157 (July-September 2000): 348-61. Este punto de vista es contrario al principio de sola Scriptura y es rechazado por Stanton L. Jones y Mark A. Yarhouse, "The Use, Misuse, and Abuse of Science in the Ecclesiastical Homosexuality Debates", en Homosexuality, Science, 120; y Christopher Seitz, "Sexuality and Scripture's Plain Sense: The Christian Community and the Law of God", en Homosexuality, Science, 177-96.

${ }^{27}$ Paul G. Crowley, "Homosexuality and the Counsel of the Cross: A Clarification", Theological Studies 69 (2008): 637.

${ }^{28} \mathrm{Cf}$. Dan O. Via y Robert A. J. Gagnon, Homosexuality and the Bible: Two Views (Minneapolis: Fortress Press, 2003), 29. Via declara: "He intentado mostrar que si miramos a varios temas bíblicos a la luz del conocimiento y la experiencia contemporánea, podemos justificablemente hacer caso omiso de las condenaciones bíblicas incondicionales de la práctica homosexual". Ibíd., 38. Véase, Soards, Scripture and Homosexuality, 55, 64. 
que no puede ser completamente controlado. ${ }^{29}$

Consecuentemente, los defensores de este punto de vista no tienen problema con las relaciones sexuales premaritales, ${ }^{30}$ el divorcio y el nuevo casamiento, ${ }^{31}$ el adulterio, e incluso a veces con la poligamia y el incesto. ${ }^{32}$ Los representantes de una lectura evolutiva de la Escritura no solo niegan la creación directa de Dios y el orden de la creación, sino también la caída. Ellos afirman que Dios ha creado a los homosexuales y, como tal, la homosexualidad es un don de Dios, no una consecuencia de la condición caída de la humanidad. ${ }^{33}$

\section{Presuposiciones adventistas del séptimo día}

Oficialmente, los adventistas del séptimo día creen que la Escritura fue dada mediante inspiración divina y que es la revelación infalible de la voluntad de Dios. Las Escrituras "son la norma del carácter, el criterio para evaluar la

${ }^{29}$ Ronald M. Springett enfatiza "que los seres humanos pueden controlar y, por lo tanto, son responsables de su expresión sexual”. Springett, Homosexuality, 25. Ellos tienen elección. Cf. Cole, "Biology, Homosexuality", 360.

${ }^{30}$ David R. Larson declara: “El lineamiento de 'nada antes' y 'todo después' no es realista ni sabio... No deberíamos preguntarnos si permitimos que existan las uniones homosexuales y heterosexuales románticas o no; pues ya existen... Deberíamos hacer todo lo que podamos para sostenerlas y apoyar a las personas que están en ellas... También deberíamos buscar maneras de honrarlas en ceremonias cristianas apropiadas”. David R. Larson, "Christian Sexual Norms Today", en Christianity and Homosexuality, parte $5-13$.

${ }^{31}$ Rogers, Homosexuality, 43, 44.

${ }^{32}$ Véase Rogers, Homosexuality, 82. Treloar menciona a Regina Schwartz como diciendo que hay "un aborrecimiento bíblico virulento hacia el incesto, el cual resuena en lo que ella describe como la histeria bíblica general y su horror explícito acerca de la homosexualidad”. Regina Schwartz, The Curse of Cain: The Violent Legacy of Monotheism (Chicago: University of Chicago Press, 1997), 107. Citado en Treloar, “Come Out”, 51.

33Véase Rogers, Homosexuality, 81. 
experiencia, la revelación definitiva de las doctrinas, un registro fidedigno de los actos de Dios realizados en el curso de la historia"34 (Is 8:20; 66:2).

Aunque fue escrita por seres humanos, no es solo la palabra de humanos, sino también la Palabra de Dios. Los principios de interpretación deben ser derivados de la Escritura y no deberían ser impuestos sobre ella. Las deducciones de los campos de la filosofía, la psicología y la sociología que contradicen la Escritura deben ser rechazadas. Además, no se debe permitir que ni la tradición ni las ciencias naturales determinen asuntos de fe. La Escritura debe ser su propio intérprete. Hay concordancia, armonía y claridad en la Escritura. Los textos claros arrojan luz sobre los textos difíciles. El Espíritu Santo es necesario en el proceso de interpretación, pero Él no anula las revelaciones previas. ${ }^{35}$

¿Qué quiere decir todo esto? Que el asunto real en el debate sobre la homosexualidad es la naturaleza, la autoridad y la interpretación de la Escritura. ${ }^{36}$ Es claro que "la decisión que uno toma sobre la validez del comportamiento homosexual... efectivamente es una decisión sobre la auto-

${ }^{34}$ Iglesia Adventista del Séptimo Día, Asociación General, Manual de la Iglesia, 19va rev. (Buenos Aires: Asociación Casa Editora Sudamericana, 2015), 158.

${ }^{35}$ Para más detalles sobre todos estos puntos, véase Ekkehardt Mueller y Richard M. Davidson, Cómo interpretar la Biblia en diez pasos, trad. Joel Iparraguirre (Ciudad de México: GEMA Editores, 2017).

${ }^{36} \mathrm{Cf}$. Rogers, Homosexuality, 1-65; Helminiak, What the Bible Really Says, 29-41; Soards, Scripture and Homosexuality, 1-14; Via y Gagnon, Homosexuality and the Bible, 2; Wink, "Homosexuality and the Bible", 33. Cf. James R. White y Jeffrey D. Niell, The Same Sex Controversy (Minneapolis: Bethany, 2002), 15; Jack Rogers, "Presbyterian Guidelines for Biblical Interpretation: Their Origin and Application to Homosexuality", Biblical Theological Bulletin 37, no. 4 (2007): 179. 
ridad de la Biblia en la vida de la iglesia". ${ }^{37}$ Estar conscientes de las presuposiciones propias ayuda a ser consistente y evitar escollos en nuestra interpretación de los textos bíblicos.

\section{Jesús y la homosexualidad}

Aunque Jesús no realizó una declaración directa sobre la homosexualidad, su posición elevada de la Escritura y del tema es reconocible. ${ }^{38}$

1. Jesús y la ley. Jesús no abolió la ley sino señaló su verdadero significado y sus implicaciones. El sermón del Monte de Mt 5 contiene una larga sección en la que se discute la ley. En Mt 23:23, Jesús habla de los "asuntos más importantes de la ley" sin dejar de lado el principio del diezmo. Por otro lado, Robert A. J. Gagnon hace un interesante comentario sobre Mr 7:

El dicho de Marcos 7:15-19, respecto a lo que contamina a una persona, se cita a menudo como prueba de que Jesús abolió las leyes de la comida. Es más probable que Jesús pretendiera un contraste usando la figura literaria de la hipérbole. Lo que realmente importa no es lo que entra en una persona sino lo que sale... Si Jesús no abrogó ni siquiera las leyes alimentarias ni del diezmo, entonces es imposible que hubiese anulado una prescripción de inmoralidad sexual como sería la de las relaciones sexuales entre hombres. ${ }^{39}$

${ }^{37}$ Soards, Scripture and Homosexuality, 73.

${ }^{38}$ Robert A. J. Gagnon ha dedicado varias páginas a Jesús y la cuestión de la sexualidad. Via y Gagnon, Homosexuality and the Bible, 68-74; Donald J. Wold dedicó un capítulo entero a "Cristo y el homosexual". Donald J. Wold, Out of Order: Homosexuality in the Bible and the Ancient Near East (Grand Rapids, MI: Baker, 1998), 161-75.

${ }^{39}$ Via y Gagnon, Homosexuality and the Bible, 69. 
2. Jesús y la sexualidad. Jesús no toleraba las formas de relación sexual excepto la que se da únicamente dentro del matrimonio entre un hombre y una mujer. Aunque se mezcló con los pecadores y se preocupó por ellos, no toleró su comportamiento. Esto es muy claro en los casos de las tres mujeres que habían cometido pecados sexuales: la mujer pecadora que lo ungió (Lc 7:36-50), la mujer samaritana con sus diferentes compañeros (Jn 4), y la mujer hallada en adulterio (Jn 8:3-11). "Vete y no peques más", fueron sus palabras. Sus vidas cambiaron. Las prostitutas le creyeron y entrarán al reino de Dios (Mt 12:31-32). En el sermón del Monte hay dos antítesis que tratan sobre asuntos sexuales. Jesús hizo hincapié en la ley. El adulterio no comenzaría con el acto sexual, sino al pensarlo. La ley del divorcio, por otro lado, es abolida y el divorcio y el nuevo casamiento no son más opciones al menos que haya adulterio por parte de uno de los cónyuges. ${ }^{40}$ En Mt 19:18 y Mr 10:19, nuevamente Jesús confirma el séptimo mandamiento.

3. Jesús y la homosexualidad. En Mt 19 y Mr 10, Jesús tuvo una discusión con los fariseos sobre el tema del divorcio. En ese contexto, se remite al relato de la creación y cita Gn 1:27 y 2:24. Dos seres humanos, hombre y mujer, se convierten en una sola carne. Se subraya la unidad, pero una unidad que consiste en un matrimonio entre un marido y una esposa. ${ }^{41}$ En el texto hebreo, falta el término "dos". No obstante, este se encuentra en la Septuaginta. Al subrayar

${ }^{40}$ Para más información al respecto, véase Richard M. Davidson, "El divorcio y el nuevo casamiento en el Antiguo Testamento" y Ekkehardt Mueller, "El divorcio y el nuevo casamiento en el Nuevo Testamento", en El matrimonio: Aspectos bíblicos y teológicos, ed. Ekkehardt Mueller y Elias Brasil de Souza (Doral, FL: Asociación Publicadora Interamericana, 2015), 185-210 y 211-61, respectivamente.

${ }^{41}$ Véase Frank M. Hasel, "El matrimonio en la Biblia", en El matrimonio, 15-42. 
que solo dos seres humanos de sexos opuestos se convierten en uno, Jesús rechazó tanto la poligamia como la homosexualidad. Obviamente, para Jesús el relato de la creación no solo fue descriptivo. Era también prescriptivo. En Mt 19:12, Jesús habla de tres grupos de eunucos: (1) los que son desde su nacimiento, ${ }^{42}(2)$ los que fueron hechos eunucos a causa de los hombres y (3) los que se han hecho a sí mismos eunucos por causa del reino de los cielos. Probablemente el último grupo no hace referencia a eunucos literales, sino a personas como Juan el Bautista que permanecieron solteros por el bien de su ministerio. Esto implicaría que los seres humanos tienen la capacidad de posponer relaciones sexuales indefinidamente, algo cierto para heterosexuales y también para aquellos que tienen inclinaciones homosexuales.

Solo hay dos alternativas, de acuerdo a Mt 19:1-12: estar casado con una persona del sexo opuesto o permanecer soltero. Como el divorcio no es una opción, la homosexualidad tampoco lo es. En Mr 7, Jesús menciona que del corazón viene el mal, y entre otros pecados que se mencionan, hay tres transgresiones sexuales: porneía (fornicación), moicheía (adulterio) y asélgeia (lascivia, sensualidad). ${ }^{43}$ El tér-

${ }^{42}$ Algunos intentan encontrar una referencia a la homosexualidad en conexión con los eunucos de nacimiento. Jack Rogers, Jesus, the Bible, and Homosexuality: Explode the Myths, Heal the Church, ed. rev. y aumentada (Louisville, KY: Westminster John Knox Press, 2009), 78-9, refiere a M. Nissinnen, quien "sugiere que en nuestro contexto contemporáneo aquellos que son eunucos desde el vientre de su madre podrían ser considerados como personas homosexuales por el simple hecho de carecer de deseo sexual hacia personas del sexo opuesto". Esta declaración parece estar cuidadosamente elaborada al no afirmar que en los tiempos bíblicos el término "eunuco" incluía a los homosexuales. El énfasis parece caer sobre el "contexto contemporáneo" en el que algunas personas quisieran incluir a los homosexuales dentro del concepto de eunuco. En este caso, sin embargo, "eunuco" debe haberse entendido en un sentido figurado.

${ }^{43}$ Wold, Out of Order, 167-70, muestra que asélgeia puede incluir ho- 
mino griego porneía tiene un abanico de significados, y el de homosexualidad no es la excepción.

Ningún judío del primer siglo hubiese hablado de porneía (inmoralidades sexuales) sin tener en cuenta las ofensas prohibidas de Levítico 18 y 20 -especialmente el incesto, el adulterio, las relaciones sexuales entre personas del mismo sexo y la zoofilia-. ${ }^{44}$

Jesús se preocupó por guardar los mandamientos y exhibir un estilo de vida que respete lo que enseña la Biblia, y esto también incluye relaciones sexuales apropiadas. Aunque no lo dijo de manera directa, Jesús aborda el tema de la homosexualidad y la rechaza.

\section{La posición de la Iglesia Adventista del Séptimo Día}

La Iglesia Adventista del Séptimo Día ha formulado declaraciones que tratan el problema de la homosexualidad. En un documento votado en uno de los Concilios Anuales de la Asociación General, la iglesia afirma:

Los adventistas creen que la intimidad sexual es adecuada únicamente en la relación conyugal entre un hombre y una mujer. Este fue el designio establecido por Dios en la creación. Las Escrituras declaran: "Por tanto, dejará el hombre a su padre y a su madre, y se unirá a su mujer, y serán una sola carne" (Gén 2:24, NIV). En todas las Escrituras, se afirma este modelo heterosexual. La Biblia no consiente actividades o relaciones homosexuales. Los actos sexuales

mosexualidad.

44Via y Gagnon, Homosexuality and the Bible, 73. 
fuera del círculo del casamiento heterosexual están prohibidos (Lev 18:5-23, 26; Lev 20:7-21; Rom 1:24-27; 1 Cor 6: 9-11). Jesucristo afirmó la intención de la creación divina... Por estas razones, los adventistas se oponen a las prácticas y relaciones homosexuales. ${ }^{45}$

Existe otra declaración que fue votada el 4 de marzo de 2004, y habla acerca de la unión de dos personas del mismo sexo. Esta declaración es una reafirmación del matrimonio cristiano en el contexto del continuo debate en varios países que discuten si este tipo de uniones deben ser consideradas iguales y deben tener o no los mismos derechos y privilegios que los matrimonios de parejas heterosexuales. Aunque el documento trata principalmente de la familia y el matrimonio, también dedica bastante espacio al tema de la homosexualidad:

La homosexualidad es una manifestación del desorden y la fractura en las inclinaciones y relaciones humanas causados por la entrada del pecado en el mundo...

Sostenemos que todas las personas, independientemente de su orientación sexual, son amadas por Dios. No aprobamos que se señale a ningún grupo para hacerlo objeto de burlas o escarnio, y menos de abuso. Sin embargo, la Palabra de Dios, que trasciende el tiempo y la cultura, no permite un estilo de vida homosexual. La oposición de la Biblia a las uniones o a los matrimonios entre personas del mismo sexo se encuentra anclado en el plan de Dios de la creación para el matrimonio (Gén 1:26-28; 2:20-24), en la legislación divina (Lev 18:22; 20:13; 1 Cor 6:9-11) y en la

45"Homosexuality", Declaración oficial de la Iglesia Adventista del Séptimo Día, consultado el 20 de enero de 2016, https://www.adventist.org/en/ information/official-statements/statements/article/go/-/homosexuality/. Esta declaración fue votada originalmente el 3 de octubre de 1999, y fue revisada por el Comité Ejecutivo de la Asociación General el 17 de octubre de 2012. 
confirmación explícita de Jesús de una relación matrimonial permanente, monógama y heterosexual (Mat 19:4-6). ${ }^{46}$

La posición de la iglesia sobre el tema de la homosexualidad es clara. La iglesia se opone a cualquier actividad homosexual y basa sus declaraciones y decisiones en la Biblia. Asimismo, la iglesia no acepta sindicatos, grupos o asociaciones homosexuales aun cuando estas puedan estar aprobadas en diferentes naciones a nivel mundial y su estatus pueda ser igual o similar a los matrimonios heterosexuales.

Incluso si estas uniones homosexuales se llaman matrimonios y las personas involucradas tienen un certificado matrimonial emitido por un gobierno, la iglesia no lo reconoce ni lo acepta. La iglesia, sin embargo, reconoce las decisiones gubernamentales siempre y cuando estén de acuerdo con la Biblia y no la contradigan.

\section{Los adventistas y el ministerio hacia los homosexuales}

¿Cómo, entonces, deberían los adventistas ministrar a los homosexuales?

1. Respeto. Los adventistas respetan a todas las personas, tanto heterosexuales como homosexuales. Reconocen que todos los seres humanos han sido creados y son valiosos a la vista de Dios. Por lo tanto, los adventistas se niegan a odiar, despreciar o abusar de los homosexuales. Aunque no toleran el pecado de la homosexualidad -como cualquier otro pecado - tratan a cada individuo con respeto sabiendo que todas las personas son pecadoras y dependen

46“Same-Sex Unions”, Declaración oficial de la Iglesia Adventista del Séptimo Día, consultado el 20 de enero de 2016, https://www.adventist.org/en/ information/official-statements/statements/article/go/-/same-sex-unions/. 
de la gracia de Dios, aunque también están llamadas a servir a Cristo y a separarse del pecado.

2. Prevención. Joseph y Linda Ames Nicolosi sugieren que, en muchos casos, la homosexualidad podría ser prevenida y la mayoría de los padres "preferirían que sus hijos [pequeños] no se involucren en conductas homosexuales". ${ }^{47}$ Los problemas de identidad de género pueden surgir en la infancia. Pero "no existe tal cosa como un 'niño gay' o un 'adolescente gay'. Todos hemos sido diseñados para ser heterosexuales. La confusión sobre el género es ante todo una condición psicológica y, en cierto modo, puede ser modificada". Esto quiere decir que el problema puede ser resulto "con o sin intervención psiquiátrica"..$^{4} \mathrm{El}$ papel de los padres es crucial. La iglesia puede ayudar a los padres a través del departamento de Ministerio de la Familia y con programas que fomenten la identidad sexual en sus hijos a fin de impedir que se desarrolle una orientación homosexual más adelante.

3. Cuidado. Aunque el siguiente párrafo se dirige específicamente a los pastores, también se puede aplicar a los miembros de iglesia que de alguna manera notan parejas homosexuales o actividad homosexual en otros miembros.

A los pastores y miembros de iglesia que se den cuenta de estos problemas, es decir, de gente que mantienen y/o promueven un estilo de vida homosexual, se les pide que sean capaces

${ }^{47}$ Joseph y Linda Ames Nicolosi, A Parent's Guide to Preventing Homosexuality (Downers Grove, IL: InterVarsity, 2002), 12.

${ }^{48} \mathrm{Ibíd}$., 16. Este es un tema que desafía la corrección política de nuestros tiempos, ya que es un tabú hablar de cambiar o dar forma a la orientación sexual de los niños en algunos lugares. Los Nicolosi, señalan que "antes de la decisión de la Asociación Americana de Psiquiatría [APA, por sus siglas en inglés] en 1973, era prácticamente aceptado tratar de prevenir la homosexualidad. Esta condición era un trastorno, y el desarrollo desordenado de la identidad sexual debía ser evitado siempre que sea posible. Hoy creemos que es hora de volver a examinar esta idea de prevención”. Ibíd., 14. 
de amarlos como para cuidar de su bienestar y visitarlos con el objetivo de iniciar un diálogo sin prejuicios y ganar sus corazones. Escuchar es crucial en todas las etapas del proceso de comunicación, especialmente al principio. Los homosexuales no necesitan un "sermón" sobre su comportamiento pecaminoso. Necesitan que una persona esté dispuesta a estar cerca y abierta para escuchar lo que tenga que decir. La confrontación sensible se reserva para un tiempo posterior después de que la relación inicial se haya establecido. Este aspecto, normalmente, está fuera de lugar durante las primeras visitas.

Las personas deben tener la oportunidad de hablar sobre sus alegrías y tristezas, así como también sobre sus sentimientos de frustración, rechazo, culpa y vergüenza. Algunos pueden saber que su comportamiento contradice la voluntad de Dios, y pueden estar abiertos a explorar nuevas direcciones para sus vidas.

En la mayoría de los casos, sin embargo, es probable que los pastores y sus ayudantes no estén capacitados para evitar una confrontación directa. Esto se debe a que la sociedad en general nos invita a que rechacemos a los homosexuales, y muchas veces este sentimiento se traslada y se comparte entre los miembros de iglesia. Pero nosotros no podemos comportarnos igual. Aunque no hay reglas específicas para acercarnos a los homosexuales, será necesario una atención y un cuidado especial, y esto debe ir acompañado por un espíritu sin prejuicios. Además, será una valiosa oportunidad para compartir textos bíblicos pues puede haber una falta de conocimiento de la Biblia por parte del miembro de iglesia que está en falta.

Muchas veces el cuidado y el asesoramiento pastoral ocurren en tres etapas: (1) Investigar la situación desde la perspectiva del que está en falta. Los sentimientos y la comprensión de la situación son importantes para el pastor. (2) Ayudar al miembro de iglesia que está en falta a desarrollar 
nuevas perspectivas y metas. (3) Formular y llevar a cabo, con el apoyo del pastor, programas de acción elegidos por el miembro de iglesia en falta, siempre y cuando estos programas no contradigan sino correspondan con las enseñanzas de la Biblia.

Si a pesar de todo el cuidado de la iglesia el miembro en falta continúa participando y/o promoviendo cualquier actividad o estilo de vida homosexual, será necesario seguir los pasos esbozados por Jesús en Mt 18:15-20. El objetivo debe ser siempre amar al pecador e intentar ganarlo. Este enfoque forma parte del cuidado pastoral.

4. Cambio. 1 Corintios 6:9-10 es uno de los textos clásicos del NT sobre el tema bajo discusión. ${ }^{49}$ A través de su contexto, se puede concluir que la homosexualidad descrita en el v. 9 no se limita a ciertas formas de actividad homosexual. Sin embargo, usualmente se deja de lado la discusión del v. 11: "Y esto erais algunos; mas ya habéis sido lavados, ya habéis sido santificados, ya habéis sido justificados en el nombre del Señor Jesús, y por el Espíritu de nuestro Dios”. Pablo, después de haber presentado su lista de vicios, concluye señalando de manera general a algunos miembros de la iglesia de Corinto que han estado involucrados en estos actos despreciables, incluyendo el pecado de la homosexualidad, pero que al final han experimentado un cambio. No son más fornicarios, idólatras, adúlteros, ladrones, borrachos, homosexuales, etc. que no heredarán el reino de Dios. iSe han salvado! Por lo tanto, cambiar es posible, pues "una conversión genuina cambia las tendencias al mal hereditarias y cultivadas". ${ }^{50}$

Si existen ministerios que brindan ayuda a los homosexuales en el proceso de cambio como Desert Stream, Exo-

${ }^{49}$ Más detalles en Mueller, Homosexualidad, la Biblia y la iglesia, 51-5.

${ }^{5}$ Elena G. de White, Maranata: El Señor viene (Doral, FL: Asociación Publicadora Interamericana, 2008), 245. 
dus International, Homosexuales anónimos, Courage, OneByOne y Regeneración, ${ }^{51}$ ¿por qué los pastores y miembros de iglesias adventistas no podrían asistir a homosexuales que desean cambiar? ${ }^{52}$

5. Ayuda. Aunque las iglesias locales pueden recurrir a la disciplina eclesiástica ${ }^{53}$ como último recurso para ayudar a los miembros de iglesia que están pecando permanentemente, que no se arrepienten y/o promueven un estilo de vida homosexual, los adventistas apoyan a aquellos que están luchando para lograr un cambio.

Jesús afirmó la dignidad de todos los seres humanos y extendió su mano compasivamente a personas y familias que sufren las consecuencias del pecado. Ofreció un ministerio cariñoso y palabras de consuelo a personas que luchan, haciendo diferencia entre su amor por los pecadores, y sus claras enseñanzas sobre las prácticas pecaminosas. Como sus discípulos, los adventistas del séptimo día se esfuerzan por seguir la instrucción y el ejemplo del Señor, viviendo una vida de compasión y fidelidad semejante a la de Cristo. ${ }^{54}$

${ }^{51} \mathrm{Cf}$. Robert A. J. Gagnon, The Bible and Homosexual Practice: Texts and Hermeneutics (Nashville, TN: Abingdon Press, 2001), 422-23.

${ }^{52} \mathrm{Entre}$ los autores que apoyan la idea de que es posible un cambio, véase Samuel Koranteng-Pipim, Must we be Silent? Issues Dividing Our Church (Ann Harborm, MI: Berean Books, 2001), 70-1; Jeffrey Satinover, Homosexuality and the Politics of Truth (Grand Rapids, MI: Baker, 1998), 245-46; Wold, Out of Order, 205.

53Para mayor información sobre cómo tratar con los miembros que yerran y las razones por las que los miembros pueden ser disciplinados, véase el capítulo 7 sobre disciplina en el Manual de la Iglesia, 55ss.

54"Homosexuality", Declaración oficial de la Iglesia Adventista del Séptimo Día, consultado el 20 de enero de 2016, https://www.adventist.org/en/ information/official-statements/statements/article/go/-/homosexuality/. 


\section{Conclusión}

Los adventistas del séptimo día entienden que la Biblia es la Palabra inspirada de Dios y revela su voluntad. Además, esta es el estándar que supera nuestra razón, emociones, experiencias y tradiciones. Nos negamos, por lo tanto, a reinterpretar la Biblia a fin de ajustarla a nuestros deseos personales o al contexto filosófico actual aún si esto produce impopularidad o consecuencias indeseables.

¿Adónde, entonces, debemos ir como iglesia? Köstenberger, profesor de NT en el Seminario Teológico Bautista del Sureste en Estados Unidos, sugiere lo siguiente para la iglesia cristiana en general, que bien podría aplicarse también para la Iglesia Adventista.

Sin duda, la clara proclamación de la iglesia de la enseñanza bíblica sobre la homosexualidad debe acompañarse con la proclamación del amor de Dios hacia todas las personas, incluyendo los homosexuales.... La homosexualidad no es el pecado imperdonable y el perdón se encuentra siempre disponible (1 Cor. 6:11). Sin embargo el perdón implica arrepentimiento y el arrepentimiento implica admisión del error. ${ }^{55}$

Ekkehardt Mueller muellere@gc.adventist.org Instituto de Investigaciones Bíblicas Asociación General de los adventistas del séptimo día

Recibido: 15/02/2017

Aceptado: 10/04/2017

${ }^{55}$ Andreas J. Köstenberger, God, Marriage, and Family: Rebuilding the Biblical Foundation (Wheaton, IL: Crossway, 2004), 223. Cursiva en el original. 